\title{
Relación entre plasticidad y ejecución cognitiva: el potencial de aprendizaje en ancianos con deterioro cognitivo
}

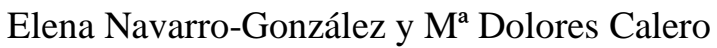 \\ Universidad de Granada (España)
}

\begin{abstract}
La vejez es una fase amplia de la vida caracterizada por importantes diferencias interindividuales y trayectorias vitales diferentes que han sido sintetizadas en tres modalidades de envejecimiento: usual, patológico y con éxito (Rowe y Khan, 1997). El estudio de las variables asociadas a dichos patrones y a la evolución cognitiva en la vejez ha generado numerosos estudios, entre los cuales destacamos el análisis del potencial de aprendizaje o la plasticidad cognitiva como herramienta evaluativa que permite establecer diferencias entre ancianos a un nivel mayor del que permiten las evaluaciones estáticas (Schreiber y Schneider, 2006). El objetivo de esta investigación es analizar en una muestra inicial de 312 ancianos con perfiles cognitivos diferentes el rendimiento en la prueba de evaluación del potencial de aprendizaje AVLT-PA para determinar en qué medida diferentes marcadores de la misma sirven para clasificar a los participantes de la manera más correcta y cuáles sirven para predecir la evolución cognitiva de los ancianos a largo plazo. Los resultados obtenidos muestran que el marcador más idóneo de la prueba AVLT-PA para clasificar a los ancianos y determinar su evolución cognitiva es la medida de recuerdo demorado de la prueba.
\end{abstract}

Palabras clave: Plasticidad cognitiva, envejecimiento exitoso, AVLT-PA, DCL.

Relationship between plasticity and cognitive performance: the potential of learning in cognitively impaired elderly. Old age is an extended period of life characterized by major inter-individual differences and different life trajectories which have been synthesized in three modalities of ageing: usual, pathological and successful (Rowe and Khan, 1997). Analysis of the variables associated with these patterns and with cognitive evolution in old age has generated a large number of studies, many of which involve the analysis of learning potential or cognitive plasticity as an assessment tool which enables us to establish differences between old adults with greater precision than static assessments (Schreiber and Schneider, 2006). The present study analysed the performance of an initial sample of 312 old adults with different cognitive profiles in the AVLT-PA learning potential assessment task, with the objective of determining which components of the task may be used to classify participants most correctly, and which may be used to predict the long-term cognitive evolution of old adults. Results indicate that the most appropriate marker of the AVLT-PA task for classifying old adults and determining their cognitive evolution is the Delayed Recall measure.

Key words: Cognitive plasticity, successful ageing, AVLT-LP, MCI.

Correspondencia: Elena Navarro González. Facultad de Psicología. Universidad de Granada, Campus de Cartuja, s/n. 18071 Granada (España). E-mail: enavarro@ugr.es 
La vejez es una fase amplia de la vida caracterizada por importantes diferencias inter individuales y con trayectorias vitales diversas que implican en algunos casos altos niveles de funcionamiento cognitivo y físico (envejecimiento exitoso), y en otros casos la presencia de altos niveles de dependencia y deterioro (envejecimiento patológico) y que ha sido sintetizada por autores como Rowe y Khan (1997) en tres modalidades de envejecimiento: usual, patológico y con éxito. Dicha diversidad en los patrones de envejecimiento ha generado en los últimos años importantes líneas de investigación dirigidas, entre otros aspectos, a clarificar qué variables bio-psico-sociales se asocian a los distintos patrones de envejecimiento. En cuanto al envejecimiento exitoso podemos señalar que los estudios epidemiológicos indican que un estilo de vida activo y rico a nivel mental, físico y social parecen proteger contra el declive cognitivo y la demencia (Fratiglioni et al., 2004; Lövden et al., 2005) aunque aún no están claros cuales son los mecanismos a partir de los cuales dichos factores influyen sobre el envejecimiento (Lövden et al., 2010). En el ámbito del envejecimiento exitoso podemos señalar un estudio reciente de Fernández-Ballesteros et al. (2010) en el que encuentran que los mejores predictores del envejecimiento exitoso son el género masculino, la alta renta, el nivel educativo alto, la menor edad, la dieta mediterránea, la actividad física, la fuerza y la amplitud pulmonar y el mejor rendimiento en una tarea de aprendizaje y coordinación psicomotriz: dígitos-símbolos del WAIS (Fernández-Ballesteros et al., 2010).

Una fase intermedia entre el envejecimiento normal y la demencia, cuando se parte de la hipótesis de un continuo cognitivo, la constituye el constructo denominado deterioro cognitivo leve (DCL) definido por Petersen et al. (2001) como una fase previa a la demencia de tal manera que si bien todas las personas que acaban desarrollando demencia, en concreto demencia tipo Alzheimer (DTA), han pasado por dicha fase, no todas las personas con DCL acaban desarrollando demencia (Nelson y O'Conner, 2008). El DCL se caracteriza por déficits cognitivos -en especial de memoria- que superan a los esperables por la edad y el nivel educativo de la persona y que pueden dar lugar a ciertas dificultades en la vida diaria aunque el sujeto no cumple los criterios de demencia (Petersen, 2003). Numerosos estudios se han llevado a cabo para intentar determinar qué variables se asocian a que una persona con DCL acabe desarrollando demencia y en este ámbito se ha observado que un amplio conjunto de funciones cognitivas, en concreto memoria episódica, memoria semántica, funciones ejecutivas y velocidad perceptiva, parecen sufrir un declive desde años antes de que aparezca la demencia (Albert et al., 2001; Bäckman et al., 2001; Peraita et al., 1999). No obstante, en dichos estudios se observan importantes solapamientos en las puntuaciones entre aquellos individuos que acaban desarrollando demencia y aquellos que no lo hacen (Bäckman et al., 2005) lo cual hace que en la actualidad no exista evidencia suficiente que nos permita recomendar pruebas cognitivas concretas para la identificación precoz de la demencia (Winblad et 
al., 2004). Una alternativa evaluativa que puede ayudar a tal efecto es la derivada de las investigaciones del grupo de Baltes (por ejemplo, Baltes et al., 1986, 1988; Lindenberger y Baltes, 1997) que denominan "testing the limits" (en España se denomina evaluación del potencial de aprendizaje o evaluación de la plasticidad cognitiva) que permite estimar el nivel de ejecución potencial de un sujeto en condiciones óptimas de evaluación en vez de su nivel actual de rendimiento tal y como se hace desde una aproximación estática en la evaluación psicológica (Schreiber y Schneider, 2006). En este procedimiento de evaluación se utiliza un formato de pretest -entrenamientoposttest de tal manera que la diferencia entre el posttest y el pretest se utiliza como un indicador de plasticidad cognitiva, ya que nos indica hasta qué punto el sujeto se ha beneficiado de una fase intermedia de entrenamiento (Fernández-Ballesteros et al., 2009). La plasticidad cognitiva (también conceptualizada como reserva cognitiva o potencial de aprendizaje) puede ser entendida como una expresión de la plasticidad neuronal (Baltes y Singer, 2001; Kempermann et al., 2002; Fernández-Ballesteros et al., 2007) y es definida como el rango en que un sujeto dado puede mejorar su rendimiento en una tarea cuando se le expone a condiciones de optimización de su ejecución que pueden ir desde el simple retest hasta entrenamientos cognitivos de larga duración (Raykov et al., 2002). En relación con la plasticidad cognitiva, las investigaciones han podido determinar la presencia de plasticidad en ancianos sanos (Baltes et al., 1986), las diferencias en plasticidad relacionadas con la edad (Nyberg et al., 2003; Yang, Krampe, y Baltes, 2006; Yang y Krampe, 2009), y la disminución de plasticidad en ancianos con deterioro cognitivo y sus implicaciones de cara al diagnóstico precoz de la demencia (Baltes et al., 1995, 1996, 1997; Raykov et al., 2002). En relación con este último punto, la idea de la que se parte es que en una fase pre-clínica de la demencia, los cambios estructurales en el cerebro asociados a procesos neuronales patológicos tendrían un impacto negativo sobre la plasticidad cognitiva por lo que examinarla podría ser un indicador útil de estatus cognitivo del sujeto. De los estudios que se han hecho partiendo de esta hipótesis (ver investigaciones de Baltes et al., 1995, 1996, 1997; Raykov et al., 2002) las conclusiones principales son dos: 1) los ancianos con riesgo de demencia se benefician menos de los programas de entrenamiento cognitivo (lo cual indicaría una menor plasticidad cognitiva); y 2) las puntuaciones de plasticidad cognitiva magnifican las diferencias inter-individuales a un nivel mayor de lo que es posible cuando se toman sólo puntuaciones de línea base (pre-test) tal y como se hace cuando se lleva a cabo una evaluación estática (Schreiber y Schneider, 2006).

En un estudio reciente Lövden et al. (2010) proponen una herramienta teórica de trabajo para el estudio de la plasticidad cognitiva en la vejez en la que diferencian entre dos términos: plasticidad y flexibilidad. La flexibilidad es entendida por los autores como el rango potencial de rendimiento que tiene una persona en un momento dado y hace referencia a la capacidad para optimizar el rendimiento del cerebro dentro de los 
límites de su estado actual de funcionamiento, mientras que la plasticidad requeriría además -según los autores- un cambio a nivel estructural y sólo se podría hablar de la misma cuando los cambios funcionales se acompañaran de dichos cambios estructurales (Lövden et al., 2010). En este sentido, según estos autores, las mejoras en el rendimiento en una tarea concreta que puedan atribuirse a la práctica o al aprendizaje relacionado con dicha tarea deberían considerarse como evidencias de flexibilidad pero no de plasticidad como se plantea en otros estudios revisados con anterioridad. Para que hubiera plasticidad debería -según Lövden et al. (2010)- darse transferencia, esto es, debería haber mejora en tareas no directamente entrenadas.

Las tareas de memoria han sido frecuentemente usadas como medida de plasticidad en ancianos, probablemente debido a que constituye una de las áreas más afectas en el DCL y la demencia. En los trabajos realizados se ha mostrado una mejora significativa en ancianos sanos y con DCL tras entrenamientos en memoria (ver metaanálisis de Zehnder et al., 2009; Martin et al., 2011) y un mantenimiento de las mejoras durante meses, e incluso años ( $\mathrm{Li}$ et al., 2008) aunque escasa transferencia a otras tareas, efecto que podría relacionarse con el tipo de entrenamiento que se suministra centrado generalmente en tareas de memoria, y que se basa en el control activo, incremento atencional y agrupamiento de los estímulos en categorías.

Nuestro grupo de investigación lleva más de 15 investigando en el ámbito del potencial de aprendizaje en la vejez en ancianos sanos y con deterioro cognitivo utilizando para ello una tarea de memoria verbal auditiva adaptada a la metodología de evaluación del potencial de aprendizaje (AVLT-LP, Wiedl et al., 1999, adaptada al español por Calero, 2000) en la cual se sigue un formato de pretest-entrenamientoposttest. Hasta ahora, de entre las posibles medidas de mejora en la prueba hemos utilizado como indicador de plasticidad o potencial de aprendizaje la puntuación de ganancia simple en la prueba, esto es, la medida posttest-pretest. Los resultados encontrados utilizando dicha medida han sido publicados en estudios previos y hacen referencia a la mayor plasticidad en ancianos con estilos de vida activo, diferencias en plasticidad entre ancianos mayores y menores de 80 años, y la comprobación de la capacidad predictiva de la plasticidad en cuanto a la evolución cognitiva de los ancianos (Calero y Navarro, 2004, 2007, 2011; Calero, Navarro y Muñoz, 2007; Navarro y Calero, 2009).

El objetivo de la investigación que aquí presentamos ha sido analizar en un amplio grupo de ancianos con perfiles cognitivos diferentes (ancianos con un alto nivel de funcionamiento cognitivo/ ancianos con un nivel de funcionamiento cognitivo normal/ ancianos con un nivel de funcionamiento cognitivo patológico) el rendimiento en la prueba AVLT-PA. En esta investigación vamos a analizar en qué medida diferentes marcadores de la prueba sirven para clasificar a los participantes de la manera más 
correcta y por tanto actúan como indicadores diagnósticos adecuados y cuáles sirven para predecir la evolución cognitiva de los ancianos a largo plazo.

\section{METODO}

\section{Participantes}

En esta investigación han participado un total de 317 ancianos con una edad media de 78,08 años (DT=7,97, rango de edad=65-96) de los cuales el 38,5\% eran hombres y el $61,5 \%$ eran mujeres. Atendiendo al estado civil el 44,8 \% eran viudos, el $28,8 \%$ solteros, el $26,2 \%$ casados y el $8,2 \%$ separados o divorciaos, el 31,3\% vivían en sus casas y el 68,8 \% en Residencias de Ancianos. A los dos años se ha llevado a cabo un seguimiento de 180 sujetos.

\section{Instrumentos}

1. Pruebas para la evaluación del estatus cognitivo:

Mini-Examen-Cognoscitivo (MEC, Lobo, Ezquerra, Gómez, Sala y Seva, 1979): Traducción y adaptación española del Mini-Mental-State-Examination (MMSE, Folstein et al., 1975). El MEC es un instrumento de screening ampliamente utilizado en la detección del deterioro cognitivo que explora de forma rápida y estandarizada un conjunto de funciones cognitivas (orientación temporo-espacial, memoria inmediata y a largo plazo, atención, cálculo, lenguaje, razonamiento abstracto y praxias) que pueden estar afectadas en personas mayores. La puntuación final obtenida en esta prueba se utiliza habitualmente como índice global y como método de seguimiento evolutivo de las funciones cognitivas en procesos como el deterioro cognitivo y la demencia habiéndose puesto de manifiesto la utilidad del MMSE (Petersen et al., 2001; Blesa et al., 2001; Bermejo et al., 1994) y del MEC (Ripoll, 2000; Vilalta et al., 1990, 1996) en el diagnóstico del deterioro cognitivo leve. La validez concurrente del MEC con una amplia batería neuropsicológica ha sido demostrada en un estudio previo (Calero et al., 2000).

Tarea de Fluidez verbal. Fluidez categorial (Spreen y Strauss, 1998). Tarea simple de recuerdo de palabras de una categoría dada que consiste en pedirle a la persona que diga tantas palabras como pueda de la categoría "animales". El evaluador anota las palabras válidas citadas por el evaluado durante un minuto. La fluidez verbal mide principalmente la velocidad y facilidad de producción verbal (denominación y vocabulario); además, evalúa la disponibilidad para iniciar una conducta en respuesta ante una tarea novedosa (Lezak, 1995; Parker y Crawford, 1992).

Tarea de evaluación de la memoria de trabajo de Oakhill, Yuill y Parkin (1989). Tarea que mide la amplitud de la memoria de trabajo a partir de la presentación de tarjetas con tres números cada una, de manera que la persona debe leer en voz alta los 
números de cada tarjeta y recordar el último número ya que luego tendrá que reproducirlos una vez que se le hayan presentado las tarjetas. El número de tarjetas se va incrementando en función del rendimiento de la persona de tal manera que la tarea empieza con dos tarjetas y acaba con cinco, siempre que la persona supere las fases anteriores.

Tarea de Atención Sostenida (TAS) (Calero y Salguero, 2008). Se trata de una tarea computarizada que consiste en la aparición aleatoria en intervalos de un segundo de números del 1 al 9, debiendo pulsar la barra espaciadora inmediatamente después a la aparición de la secuencia "3-6". Los estímulos numéricos aparecen en blanco sobre un fondo negro. Existe un bloque inicial de ensayos de entrenamiento y cuatro bloques posteriores de prueba. Cada bloque experimental tiene una duración de 1 minuto y en cada uno aparece aleatoriamente la secuencia "3-6" quince veces sobre 60 secuencias posibles, siendo siempre las 45 secuencias restantes consideradas como distractores. Así, al finalizar la tarea (los cuatro bloques experimentales) habrán aparecido 60 secuencias correctas sobre un total de 240 secuencias posibles. La variable dependiente en esta tarea es el número de aciertos totales por participante (número de veces que aparece la secuencia correcta y el sujeto pulsa la barra espaciadora).

CUBRECAVI. Cuestionario breve de calidad de vida (Fernández-Ballesteros y Zamarrón, 2007). El CUBRECAVI es un cuestionario dirigido a la evaluación multidimensional de la calidad de vida en personas mayores compuesto por 21 subescalas agrupadas en las siguientes escalas: 1) salud (subjetiva, objetiva y psíquica), 2) integración social, 3) habilidades funcionales, 4) actividad y ocio, 5) calidad ambiental, 6) satisfacción con la vida, 7) educación, 8) ingresos, y 9) servicios sociales y sanitarios. Cada subescala se evalúa mediante una o varias cuestiones y se ofrecen puntuaciones independientes para cada una de las anteriores escalas.

Baremo de valoración de la dependencia (BVD, Ministerio de Asuntos Sociales): Esta prueba consiste en una versión reducida del Baremo de Valoración de la Dependencia elaborado por el Ministerio de Asuntos Sociales para determinar el grado de autonomía de la persona. En este caso, se han seleccionado aquellas escalas relacionadas con la dependencia en la vejez, en concreto las siguientes: valoración en comer y beber; valoración en la regulación de la micción y defecación; valoración en lavarse; valoración en otros cuidados corporales; valoración en vestirse; valoración en mantenimiento de la salud; valoración en transferencia corporal; valoración en desplazamientos dentro y fuera del hogar; y valoración de realización en tareas domésticas. En cada escala se señalan una serie de tareas y para cada una de ellas el cuidador habitual de la persona (en nuestro caso el personal auxiliar sanitario de las Residencias) debía indicar el desempeño y en el caso de necesitar ayuda el grado de apoyo personal que requerían. En este cuestionario se obtienen puntuaciones por escalas 
y una puntuación global con el rango 0 a 100 en donde 0 indicaría independencia total y 100 dependencia total.

2. Prueba de evaluación del potencial de aprendizaje:

Test Auditory Verbal Learning Test-Potencial de aprendizaje (AVLT-PA, Wiedl et al., 1999, adaptado al español por Calero, 2000): Versión del clásico test de Memoria Verbal de Rey que consiste, en su versión tradicional, en la presentación de quince palabras comunes que el participante debe repetir inmediatamente después de haberlas escuchado. La lista se presenta un total de cinco veces y en cada ocasión se anotan las palabras que ha podido recordar el sujeto. En la versión de potencial de aprendizaje, la lista de palabras se presenta seis veces. Las dos primeras hacen de pretest y se sigue la forma de presentación estándar, las dos siguientes son de entrenamiento e incluyen retroalimentación sobre la ejecución, refuerzo y repetición de palabras no recordadas, y las dos últimas hacen de posttest y vuelven por ello a ser de presentación estándar. Una vez pasada la prueba y tras realizar el participante otra serie de pruebas cognitivas que actúan a modo de interferencia, al sujeto se le pide que de nuevo recuerde las palabras de la lista sin darle ninguna ayuda. La aplicación de la prueba con el formato expuesto permite obtener distintos marcadores. En esta investigación se ha trabajado con los siguientes:

AVLT-PA entrenamiento: puntuación que se obtiene de la media de aciertos en las dos presentaciones intermedias de la lista de palabras.

AVLT-PA post: puntuación que se obtiene de la media de aciertos en las dos últimas presentaciones de la lista de palabras.

AVLT-PA puntuación de ganancia: diferencia posttest-pretest.

AVLT-PA recuerdo demorado: número de palabras de la lista que la persona recuerda una vez terminada la tarea y tras la realización de una serie de pruebas que actúan a modo de interferencia.

\section{Procedimiento}

Los participantes en este estudio proceden de tres ámbitos diferentes tal y como se reflejó anteriormente: Residencias de ancianos, Centros de Día, y Universidad de Mayores de Granada. En los tres ámbitos, se contactó con el equipo directivo de cada Centro y se les presentó el Proyecto para pedir la colaboración en el estudio propuesto. Una vez que los Centros daban su consentimiento se les pedía que hicieran una selección de ancianos que cumplieran los siguientes requisitos: no padecimiento de enfermedades relevantes o limitaciones físicas y/o psíquicas así como ausencia de déficits motores y/o sensoriales. Una vez seleccionados los participantes, y de manera previa a la evaluación, cada participante era informado de manera individual de los objetivos del trabajo y de la evaluación a realizar para que éstos, si querían, dieran su consentimiento informado. La evaluación ha sido llevada en todos los casos por psicólogos experimentados y 
entrenados en técnicas de evaluación psicológica y de evaluación del potencial de aprendizaje. Las sesiones de evaluación consistieron en dos sesiones de 1 hora de duración máxima cada una.

Los 317 participantes iniciales han sido clasificados en tres grupos en función de las puntuaciones obtenidas en las pruebas de evaluación del estatus cognitivo (ver apartados instrumentos de evaluación) utilizando los criterios que diferentes publicaciones determinan para cada grupo (Fernández-Ballesteros et al., 2007). El grupo de envejecimiento exitoso está formado por 178 ancianos que presentan: independencia, alto nivel cognitivo (MEC por encima de 27 puntos), satisfacción con la vida, salud objetiva y subjetiva y compromiso social y productivo (Fernández-Ballesteros et al., 2007). El grupo de envejecimiento normal está formado por 95 ancianos con MEC entre 20-26, autonomía y moderadas o altas puntuaciones en salud y satisfacción con la vida, y el grupo de envejecimiento patológico está formado por 44 ancianos con MEC por debajo de 20, quejas de memoria y menores habilidades funcionales no asociadas a alteración psicopatológica o déficit sensorial importante siguiendo criterios de Petersen (2003). En el seguimiento a los dos años se ha evaluado con la prueba MEC a un total de 180 ancianos de los cuales y atendiendo a los mismos criterios de clasificación 100 presentan envejecimiento exitoso, 56 con envejecimiento normal y 26 envejecimiento patológico. El diseño seguido ha sido un diseño correlacional predictivo de caso típico. Se han realizado ANCOVAs y análisis discriminantes de las diferentes medidas del AVLT-PA respecto de los grupos establecidos.

\section{RESULTADOS}

En primer lugar, podemos señalar que, tal y como se refleja en la tabla 1, los tres grupos establecidos presentan entre ellos diferencias significativas no sólo en medidas cognitivas (fluidez verbal, memoria de trabajo, atención sostenida) sino también en dependencia, en edad y en algunas variables de calidad de vida tales como salud, integración social, nivel educativo, habilidades funcionales, actividad y ocio, satisfacción con la vida y nivel de independencia. No se observan sin embargo diferencias entre grupos en algunas de las dimensiones de la calidad de vida evaluadas con la prueba CUBRECAVI como son salud subjetiva y objetiva, calidad ambiental, calidad de vida, y uso de servicios sociales de la comunidad.

En segundo lugar, respecto a los diferentes indicadores que pueden derivarse del AVLT-PA vemos (Figura 1) cómo existen diferencias entre los tres grupos en todas los indicadores utilizados. Así, si realizamos un ANOVA entre grupos controlando la variable edad, las diferencias son significativas en todos los casos. 
NAVARRO-GONZÁLEZ y CALERO. Relación entre plasticidad y ejecución cognitiva

Tabla 1. Diferencias entre los tres grupos establecidos según criterios de envejecimiento saludable en las diferentes variables evaluadas

\begin{tabular}{|c|c|c|c|c|c|}
\hline & & $M$ & D.T. & $F$ & $p$ \\
\hline \multirow{3}{*}{ Edad } & Exitoso & 76,34 & 7,93 & & \\
\hline & Normal & 79,48 & 7,30 & 12,027 & 0,0001 \\
\hline & Patológico & 82,09 & 7,61 & & \\
\hline \multirow{3}{*}{ Salud total } & Exitoso & 3,10 & ,48 & & \\
\hline & Normal & 2,95 &, 52 & 4,801 & 0,009 \\
\hline & Patológico & 2,86 & ,64 & & \\
\hline \multirow{3}{*}{ Salud Subjetiva } & Exitoso & 2,86 &, 85 & & \\
\hline & Normal & 2,69 &, 89 & & \\
\hline & Patológico & 2,65 & ,99 & & \\
\hline \multirow{3}{*}{ Salud objetiva } & Exitoso & 3,33 & 42 & & \\
\hline & Normal & 3,24 & ,45 & & \\
\hline & Patológico & 3,25 & ,40 & & \\
\hline \multirow{3}{*}{ Salud psíquica } & Exitoso & 3,11 &, 55 & & \\
\hline & Normal & 2,92 & ,68 & 8,130 & 0,0001 \\
\hline & Patológico & 2,71 &, 77 & & \\
\hline \multirow{3}{*}{ Integración social } & Exitoso & 2,39 &, 55 & & \\
\hline & Normal & 2,16 &, 55 & 7,776 & 0,001 \\
\hline & Patológico & 2,12 &, 48 & & \\
\hline \multirow{3}{*}{$\begin{array}{l}\text { Habilidades } \\
\text { funcionales }\end{array}$} & Exitoso & 3,27 & ,79 & & \\
\hline & Normal & 2,78 & ,93 & 17,731 & 0,0001 \\
\hline & Patológico & 2,56 & ,90 & & \\
\hline \multirow{3}{*}{ Actividad y Ocio } & Exitoso & 2,33 &, 56 & & \\
\hline & Normal & 2,09 & ,49 & 13,425 & 0,0001 \\
\hline & Patológico & 1,92 & ,46 & & \\
\hline \multirow{3}{*}{ Calidad ambiental } & Exitoso & 2,99 & ,34 & & \\
\hline & Normal & 2,94 & ,45 & & \\
\hline & Patológico & 3,03 & ,28 & & \\
\hline \multirow{3}{*}{ Satisfacción vida } & Exitoso & 2,87 &, 80 & & \\
\hline & Normal & 2,76 &, 85 & 3,329 & 0,037 \\
\hline & Patológico & 2,51 & ,91 & & \\
\hline \multirow{3}{*}{ Nivel educativo } & Exitoso & 1,52 & 1,11 & & \\
\hline & Normal & 81 &, 72 & 21,323 & 0,0001 \\
\hline & Patológico & ,79 &, 79 & & \\
\hline \multirow{3}{*}{ Servicios SS } & Exitoso & 2,86 & 60 & & \\
\hline & Normal & 2,95 &, 54 & & \\
\hline & Patológico & 2,78 & ,68 & & \\
\hline \multirow{3}{*}{ Calidad de vida } & Exitoso & 1,88 &, 71 & & \\
\hline & Normal & 1,86 & ,66 & & \\
\hline & Patológico & 1,82 & 67 & & \\
\hline \multirow{3}{*}{ MEC inicial } & Exitoso & 30,59 & 2,54 & & \\
\hline & Normal & 23,78 & 2,00 & 751,895 & 0,0001 \\
\hline & Patológico & 15,77 & 2,63 & & \\
\hline \multirow{3}{*}{ Fluidez palabras } & Exitoso & 13,88 & 4,12 & & \\
\hline & Normal & 10,74 & 3,49 & 62,464 & 0,0001 \\
\hline & Patológico & 6,95 & 3,77 & & \\
\hline \multirow{3}{*}{ BVD_total } & Exitoso & 6,76 & 12,87 & & \\
\hline & Normal & 17,10 & 18,82 & 47,222 & 0,0001 \\
\hline & Patológico & 31,77 & 19,33 & & \\
\hline \multirow{3}{*}{ Atención Aciertos } & Exitoso & 43,38 & 14,65 & & \\
\hline & Normal & 31,76 & 13,84 & 27,404 & 0,0001 \\
\hline & Patológico & 27,65 & 15,48 & & \\
\hline & Exitoso & 2,45 & ,98 & & \\
\hline Memoria Trabajo & Normal & 1,37 & 1,05 & 59,157 & 0,0001 \\
\hline & Patológico &, 81 &, 84 & & \\
\hline & Exitoso & 29,17 & 4,31 & & \\
\hline MEC 2 años & Normal & 22,82 & 3,80 & 141,82 & 0,0001 \\
\hline & Patológico & 14,34 & 4,39 & & \\
\hline
\end{tabular}

Se indica $\mathrm{F}$ y $\mathrm{p}$ en aquellas variables cuya diferencia entre grupos es significativa 
Figura 1. Índices de mejora en el AVLT-LP en los tres grupos

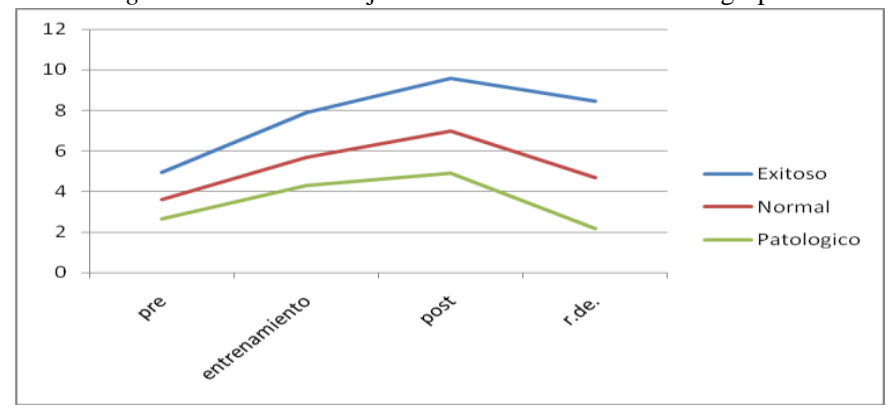

Diferencias significativas en pre $(\mathrm{F}=53,495 ; \mathrm{p}=0,0001)$; entrenamiento $(\mathrm{F}=63,987 ; \mathrm{p}=0,0001)$; post $(\mathrm{F}=69,165 ; \mathrm{p}=0,0001)$ y recuerdo demorado $(\mathrm{F}=56,895 ; \mathrm{p}=0,0001)$

Una vez llegados a este punto, pasamos a realizar un análisis discriminante por pasos incluyendo los 4 indicadores de la prueba AVLT-PA (entrenamiento, posttest, puntuación de ganancia y recuerdo demorado) respecto al grupo establecido en la evaluación inicial. Los resultados encontrados (ver tabla 2) reflejan que el recuerdo demorado, la puntuación posttest y la puntuación de ganancia, por este orden son las variables que predicen el estatus cognitivo, presentando el recuerdo demorado un autovalor de 0,557 (98,2\% de varianza).

Tabla 2. Resultados obtenidos en el análisis discriminante por pasos. V. dependiente: grupo de pertenencia según el estatus inicial

\begin{tabular}{|c|c|c|c|c|c|c|}
\hline Pasos & V. Introducidas & Tolerancia & F para salir & Wilks Lambd & $\mathrm{F}(\mathrm{g} .1)$. & $p$ \\
\hline 1 & $\begin{array}{l}\text { AVLT R. } \\
\text { demorado }\end{array}$ & 1,000 & 56,895 & ,685 & $56,895(1 / 248)$ &, 0001 \\
\hline 2 & $\begin{array}{c}\text { AVLT R. } \\
\text { demorado } \\
\text { AVLT post }\end{array}$ & $\begin{array}{l}, 312 \\
, 312\end{array}$ & $\begin{array}{l}5,288 \\
4,314\end{array}$ & ,662 & $28,249(2 / 248)$ & ,0001 \\
\hline 3 & $\begin{array}{c}\text { AVLT R. } \\
\text { demorado } \\
\text { AVLT post } \\
\text { p. ganancia }\end{array}$ & $\begin{array}{l}, 304 \\
, 162 \\
, 309\end{array}$ & $\begin{array}{l}3,710 \\
9,082 \\
5,192\end{array}$ & ,636 & $20,861(3 / 248)$ &, 0001 \\
\hline
\end{tabular}

Tabla 3. Resultados obtenidos en el análisis discriminante por pasos. V. dependiente: grupo de pertenencia según el estatus cognitivo a los dos años

\begin{tabular}{ccccccc}
\hline Pasos & V. Introducidas & Tolerancia & $\begin{array}{c}\text { F para } \\
\text { salir }\end{array}$ & Wilks Lambd & F(g.l.) & $p$ \\
\hline 1 & AVLT R. demorado & 1,000 & 28,577 &, 710 & $28,577(1 / 140)$ &, 0001 \\
\hline
\end{tabular}

Respecto del estatus cognitivo a los 2 años, la función discriminante (ver tabla 3) refleja resultados aún más contundentes, de tal manera que el recuerdo demorado 
sigue siendo la primera variable predictora con un autovalor de 0,408 (100\% de varianza).

Por último, y con el objetivo de analizar qué criterio de la prueba AVLT-PA clasifica más correctamente a los participantes de los tres grupos (envejecimiento exitoso, normal y patológico) se ha clasificado a los sujetos en función de la puntuación obtenida en el AVLT-PA. Para la clasificación en esta prueba se ha tenido lugar la puntuación media y la desviación típica obtenida por todos los participantes en los distintos apartados de la misma tanto en la evaluación inicial como en la evaluación a los dos años. Así, en el caso de la puntuación posttest se han establecido dos grupos: alta y baja puntuación con un punto de corte de 8,5 puntos (el rango es $0-15$ ); en el recuerdo demorado se han clasificado en dos grupos: alta y baja puntuación con un punto de corte de 6,5 puntos (el rango es 0-15) y en la puntuación de ganancia se han establecido dos grupos: ganadores y no ganadores con un punto de corte de 4 puntos (el rango es 0-11). Teniendo en cuenta esta clasificación y tal y como se presenta en la tabla 4, vemos que vuelve a ser el recuerdo demorado el que da cuenta mejor de las diferencias entre grupos y el que identifica mejor a los ancianos con envejecimiento patológico (tabla 4) tanto en la evaluación inicial como en el seguimiento a los dos años.

Tabla 4. Resultados de la clasificación según cada uno de los criterios utilizados (AVLT-post; recuerdo demorado y puntuación de ganancia)

\begin{tabular}{|c|c|c|c|c|c|c|c|}
\hline & & \multicolumn{2}{|c|}{ 1.- evaluación inicial } & \multicolumn{2}{|c|}{ 2.- evaluación a los 2 años } & \multirow{2}{*}{ Chi cuadrado } & \multirow{2}{*}{$p$} \\
\hline & & bajo & alto & bajo & alto & & \\
\hline \multirow{3}{*}{$\begin{array}{l}\text { AVLT- } \\
\text { posttest }\end{array}$} & Exitoso & 56 & 124 & 31 & 50 & 1.- $\quad 68,293$ & 0,001 \\
\hline & Normal & 64 & 29 & 43 & 23 & \multirow{2}{*}{ 2.- $\quad 15,730$} & \multirow{2}{*}{0,001} \\
\hline & Patológico & 40 & 4 & 32 & 3 & & \\
\hline \multirow{3}{*}{$\begin{array}{l}\text { Recuerdo } \\
\text { Demorado }\end{array}$} & Exitoso & 47 & 106 & 30 & 38 & 1.- 56,999 & 0,001 \\
\hline & Normal & 52 & 21 & 30 & 20 & \multirow{2}{*}{ 2.- } & \multirow{2}{*}{0,001} \\
\hline & Patológico & 24 & 1 & 25 & 0 & & \\
\hline \multirow{3}{*}{$\begin{array}{l}\text { AVLT- } \\
\text { puntuación } \\
\text { ganancia }\end{array}$} & Exitoso & 65 & 115 & 30 & 51 & 1.- 31,083 & 0,001 \\
\hline & Normal & 44 & 49 & 29 & 37 & \multirow{2}{*}{ 2.- 13,906} & \multirow{2}{*}{0,001} \\
\hline & Patológico & 33 & 11 & 25 & 10 & & \\
\hline
\end{tabular}

\section{CONCLUSIONES}

El objetivo del presente estudio fue analizar en un amplio grupo de ancianos con perfiles cognitivos diferentes el rendimiento en la tarea AVLT-PA con el fin de determinar en qué medida diferentes marcadores de la prueba servían para clasificar a los participantes de la manera más correcta posible y cuáles servían para predecir la evolución cognitiva de los ancianos a largo plazo. Esta idea partía en primer lugar de la clasificación existente en la literatura especializada que plantea diferentes trayectorias de envejecimiento como son envejecimiento exitoso, usual/normal, y patológico (Rowe y Khan, 1997) y de la investigación que está generando dicha clasificación en cuanto a las características bio-psico-sociales de cada tipo de envejecimiento y su evolución en el 
tiempo. En segundo lugar, y en el ámbito del estudio del potencial de aprendizaje en la vejez como posible variable relevante en este ámbito de estudio, esta investigación partía de la hipótesis propuesta por Lövden et al. (2010) según la cual las medidas simples de evaluación de la plasticidad obtenidas a partir de las ganancias tras un entrenamiento eran indicativas de flexibilidad y señalaban que las medidas de transferencia eran mejores indicadores de plasticidad. Por ello, en este estudio en primer lugar se ha clasificado a la muestra en función de su funcionamiento cognitivo y su nivel de autonomía y de salud en tres grupos: ancianos con envejecimiento exitoso, normal y patológico; y en segundo lugar se ha trabajado con distintos marcadores obtenidos a partir de la prueba AVLT-PA, tarea de memoria verbal adaptada a la evaluación del potencial de aprendizaje en la vejez de tal manera que se ha tenido en cuenta la puntuación en la fase del entrenamiento, la puntuación tras el entrenamiento, la puntuación de ganancia y el recuerdo demorado. A partir de estos parámetros se ha analizado en qué medida dichos indicadores sirven para clasificar adecuadamente a los tres grupos de participantes tanto en la evaluación inicial como en el seguimiento realizado a los 2 años.

En primer lugar, debemos señalar que los tres grupos establecidos (envejecimiento exitoso, normal y patológico) están perfectamente diferenciados, no solo en habilidades cognitivas sino también en salud, dependencia y calidad de vida de tal manera que, como se señalaba en investigaciones previas (Fernández-Ballesteros et al., 2010) los ancianos con envejecimiento exitoso son los que presentan una menor edad, una mejor salud, una mayor independencia y habilidades funcionales, un mayor nivel educativo y un nivel de ejecución cognitiva más alta.

En segundo lugar y al analizar el rendimiento de los tres grupos de ancianos en la prueba AVLT-PA y en los distintos marcadores de la misma hemos podido comprobar que existen diferencias significativas entre los tres grupos en todos los marcadores del AVLT-PA aún controlando el efecto de la edad, resultados acordes con investigaciones previas realizadas con esta prueba que muestran cómo la plasticidad cognitiva o el potencial de aprendizaje se dan en diferente cuantía según el estatus cognitivo del anciano tal y como se ha podido establecer en estudios previos (Martin et al., 2011; Zehnder et al., 2009).

En tercer lugar, los análisis discriminantes han mostrado que la puntuación de la fase de entrenamiento (que se correspondería en estricto sentido con lo que Lövden et al. (2010) consideran como flexibilidad) no es significativa para clasificar a los grupos mientras que sí lo son las medidas posttest, la puntuación de ganancia y el recuerdo demorado. En concreto, el mejor indicador es el recuerdo demorado, que podría considerarse, siguiendo el planteamiento de Lövden et al. (2010), como indicador de transferencia del entrenamiento -ya que se trataría del mantenimiento de los efectos del entrenamiento en el tiempo-, seguida por la puntuación posttest (medida de ejecución 
después del entrenamiento) y de la puntuación de ganancia (mejora de la ejecución del posttest respecto del pretest). También hemos podido observar que la capacidad discriminante del recuerdo demorado se mantiene respecto de los resultados obtenidos en el seguimiento a los dos años y que la clasificación realizada con este criterio es la más precisa ya que a partir de la misma todos los ancianos con envejecimiento patológico menos uno estarían bien clasificados en la evaluación inicial y todos estarían bien clasificados en el seguimiento a los dos años.

En definitiva, los resultados obtenidos apoyarían los planteamientos de Lövden et al. (2010) en el sentido de que corroboran la importancia de tomar medidas de transferencia (en este caso en el tiempo) además de otras medidas de plasticidad. De esta manera, en este estudio se vuelve a demostrar que el potencial de aprendizaje es un buen indicador de estatus cognitivo y un buen predictor de mantenimiento cognitivo a largo plazo. De este modo, se da más evidencia a favor de la utilización de este tipo de metodología evaluativa propuesta inicialmente por el grupo de Baltes y que ha demostrado en numerosos estudios que con la misma se magnifican las diferencias interindividuales a un nivel mayor de lo que es posible cuando se toman sólo puntuaciones de línea base (pre-test) tal y como se hace cuando se lleva a cabo una evaluación estática (Schreiber y Schneider, 2006).

No obstante, y a partir de los resultados obtenidos utilizando distintos marcadores de la prueba AVLT-PA, pensamos que pueden mejorarse los procedimientos de estimación de dicho potencial de aprendizaje si somos capaces de diseñar procedimientos que diferencien entre diferentes tipos de transferencia de los efectos del entrenamiento. Por otra parte, y teniendo en cuenta el tamaño de la muestra y el número de sujetos de los que se ha hecho seguimiento se hace necesario seguir investigando en esta línea al igual que sería interesante analizar la transferencia a otro tipo de tareas cognitivas en las que las posibilidades de entrenamiento y generalización puedan ser mejor establecidas.

\section{REFERENCIAS}

Albert, M.S., Moss, M.B., Tanzi, R. y Jones, K. (2001). Preclinical prediction of AD using neuropsychological tests. Journal of the International Neuropsychological Society, 7 , 631-639.

Bäckman, L., Small, B.J. y Fratiglioni, L. (2001). Stability of the preclinical episodic memory deficit in Alzheimer's disease. Brain, 124, 96-102.

Bäckman, L., Jones, S., Berger, A.K., Laukka, E.J. y Small, B.J. (2005). Cognitive impairment in preclinical Alzheimer's disease: A meta-analysis. Neuropsychology, 19, 520-531.

Baltes, M.M., Kuhl, K.P., Gutzmann, H. y Sowarka, D. (1995). Potential of cognitive plasticity as a diagnostic instrument: A cross-validation and extension. Psychology and Aging, 10, 167-172.

Baltes, P.B. y Lindenberger, U. (1988). On the range of cognitive plasticity in old age as a function of experience: 15 years of intervention research. Behaviour Therapy, 19, 283-300. 
Baltes, P.B. y Singer, T. (2001). Plasticity and the ageing mind: An exemplar of the bio-cultural orchestration of brain and behaviour. European Review, 1, 59-76.

Baltes, P.B., Dittmann-Kohli, F. y Kliegl, R. (1986). Reserve capacity of the elderly in agingsensitive tests of fluid intelligence: Replication and extension. Psychology and Aging, 1, 172-177.

Calero, M.D. y Navarro, E. (2004). Relationship between Plasticity, Mild Cognitive Impairment and Cognitive Decline. Archives of Clinical Neuropsychology, 19, 653-660.

Calero, M.D. y Navarro, E. (2007). Effectiveness of a memory training programme in the maintenance of status in elderly people with and without cognitive decline. Psychology in Spain, 11, 106-112.

Calero, M.D., Navarro, E. y Muñoz-Manzano, L. (2007). Influence of level of activity on cognitive plasticity in elderly persons. Archives of Gerontology and Geriatrics, 45, 307-318.

Calero, M.D. y Navarro, E. (2011). Differences in cognitive performance, level of dependency and quality of life (QoL), related to age and cognitive status in a sample of Spanish old adults under and over 80 years of age. Archives of Geriatrics and Gerontology. DOI: 10.1016/j.archger.2010.11030.

Calero, M.D. y Salguero, A. (2008) TAS: Tarea de Atención Sostenida. Granada: Sinder.

Fratiglioni, L., Paillard-Borg, S. y Winblad, B. (2004). An active and socially integrated lifestyle in late life might protect against dementia. Lancet Neurology, 3, 343-353.

Fernández-Ballesteros, R., Kruse, A., Zammarrón, M.D. y Capprara, M.G. (2007) Quality of life, life satisfaction and positive aging. En R. Fernández-Ballesteros (Ed.): GeroPsychology. European perspective for an aging world. (p. 198-223). Göttingen: Högrefe \& Huger.

Fernández-Ballesteros, R. y Zamarrón, M.D. (2007). Cubrecavi. Cuestionario breve de calidad de vida. Madrid: TEA Ediciones.

Fernández-Ballesteros, R., Zamarrón, M.D., Calero, M.D. y Tárraga, L. (2007). Cognitive plasticity and cognitive impairment. En R. Fernández-Ballesteros (Ed.): GeroPsychology. European perspective for an aging world. (p. 145-164). Göttingen: Högrefe \& Huger.

Fernández-Ballesteros, R., Zamarrón, M.D., López-Brazo, M.D., Molina, M.A., Díez, J., Montero, P., y Schettini, R. (2010). Psicothema, 22, 4, 641-647.

Kempermann, G., Gast, D. y Gage, F.H. (2002). Neuroplasticity in old age: Sustained fivefold induction of hippocampal neurogenesis by long-term environmental enrichment. Annals of Neurology, 52, 135-142.

Li, S.C., Schmiedek, F., Huxhold, O., Röcke, C, Smith, F. y Lindenberger, U. (2008). Working memory plasticity in old age: practice, gain, transfer and maintenance. Psychology and Aging, 23(4), 731-742.

Lindenberger, U. y Baltes, P. (1997). Intellectual functioning in old and very old age: first result from the Berlin aging study. Psychology and Aging, 12(1), 12-21.

Lövdén, M. y Lindenbergeru, U. (2005). Development of intellectual abilities in old age: from age gradients to individuals. En Wilhelm y Engle (eds.). Understanding and measuring intelligence (p. 203-221). Thousand Oas, CA: Sage.

Lövden, M., Bäckman, L., Lindenberger, U., Scahefer, S. y Schiedeck, F. (2010). A theoretical framework for the study of adult cognitive plasticity. Psychological Bulletin, 136, 659676.

Lobo, A., Ezquerra, J., Gómez, F., Sala, J. \& Seva, A. (1979). El Mini-Examen-Cognoscitivo. Un test sencillo y práctico para detectar alteraciones intelectuales en pacientes médicos. Actas Luso Españolas de Neurología y Psiquiatría, 19(7), 189-201. 
Martin, M., Clare, L., Algassen A.M., Cameron, M.H. y Zehnder, F. (2011). Cognition-based interventions for healthy older people with cognitive impairment. Cochrane database of systematic review (online), 1: 006220.

Navarro, E. y Calero, M.D. (2009). Estimation of cognitive plasticity in old adults using dynamic assessment techniques. Journal of Cognitive Education and Psychology, 8, 38-51.

Nelson, A.P. y O'Connor, M.G. (2008). Mild cognitive impairment: a neuropsychological perspective. CNS Spectr, 13, 56-64.

Oakhill, J., Yuill, N. y Parkin, A. (1989). Working memory, comprehension ability and the resolution of text anomaly. British Journal of Psychology, 80, 351-61.

Peraita, H., Galeote, M. y González-Labra, M.J. (1999) Deterioro de la memoria semántica en pacientes de Alzheimer: evidencia a partir de tareas de definición, clasificación y razonamiento analógico. Psicothema, 11(4), 917-937.

Petersen, R.C. (2003). Mild cognitive impairment clinical trials. Nat Rev Drug Discov, 2, 646-53.

Petersen, R.C., Doody, R., Kurz, A., Mohs, R.C., Morris, J.C. y Rabins, P.V. (2001). Current concepts in mild cognitive impairment. Arch Neurol; 58, 1985-92.

Raykov, T., Baltes, M.M., Neher, K.M. y Sowarka, D. (2002). A comparative study of two psychometric approaches to detect risk status for dementia. Gerontology, 48, 185-193.

Ripoll, A. (2000). Detección precoz del deterioro cognitivo. Psiquiatría y Atención Primaria, 1(3), 4-9.

Rowe, J.W. y Khan, R.L. (1997). Successful aging. The Gerontologist, 37, 433-440.

Schreiber, M. y Schneider, R. (2007). Cognitive plasticity in people at risk for dementia: Optimising the testing-the-limits-approach. Aging \& Mental Health, 11(1), 75-81.

Spreen, O. y Strauss, E. (1998). A compendium of neuropsychological tests: Administration, norms, and commentary (2nd ed.). New York: Oxford University Press.

Vilalta, J., Llinás, S., López-Pousas, A., Amiel, J. y Vidal, C. (1990). CAMDEX. Validación de la adaptación española. Neurología, 5, 117-120.

Wiedl, K.H., Wienöbst, J. y Schöttke, H. (1999). Estimating rehabilitation potential in schizophrenic subjects. En H.D. Brenner, W. Bokerv y R. Gennes (eds). The treatment of schizophrenia: status and emerging trends. Benn: Hografe y Hunber.

Winblad, B., Palmer, K., Kivipelto, M., Jelic, V., Fratiglioni, L., Wahlund, L.O., et al. (2004). Mild cognitive impairment: beyond controversies, towards a consensus: Report of the International Working Group on Mild Cognitive Impairment. Journal of Internal Medicine, 256, 240-246.

Zehnder, F., Martin, M., Altgassen, M. y Clare, L. (2009). Memory training effects in old age as markers of plasticity: a metaanalisys. Restorative Neurology Neuroscience, 27(5), 507520.

Recibido: 1 de julio de 2011

Recepción Modificaciones: 22 de agosto de 2011

Aceptado: 26 de septiembre de 2011 